\title{
Reversing Steps in Petri Nets
}

\author{
David de Frutos Escrig ${ }^{1}$, Maciej Koutny ${ }^{2}$, and Łukasz Mikulski ${ }^{3}$ \\ 1 Dpto. Sistemas Informáticos y Computación, Facultad de Ciencias Matemáticas \\ Universidad Complutense de Madrid, Madrid, Spain \\ defrutos@sip.ucm.es \\ 2 School of Computing, Newcastle University \\ Newcastle upon Tyne NE4 5TG, United Kingdom \\ maciej.koutny@ncl.ac.uk \\ 3 Faculty of Mathematics and Computer Science, Nicolaus Copernicus University \\ Toruń, Chopina 12/18, Poland \\ lukasz . mikulski@mat.umk .pl
}

\begin{abstract}
In reversible computations one is interested in the development of mechanisms allowing to undo the effects of executed actions. The past research has been concerned mainly with reversing single actions. In this paper, we consider the problem of reversing the effect of the execution of groups of actions (steps).

Using Petri nets as a system model, we introduce concepts related to this new scenario, generalising notions used in the single action case. We then present a number of properties which arise in the context of reversing of steps of executed transitions in place/transition nets. We obtain both positive and negative results, showing that dealing with steps makes reversibility more involved than in the sequential case. In particular, we demonstrate that there is a crucial difference between reversing steps which are sets and those which are true multisets.
\end{abstract}

Keywords: Petri net, reversible computation, step semantics

\section{Introduction}

Reversibility of (partial) computations has been extensively studied during the past years, looking for mechanisms that allow to (partially) undo some actions executed during a process, that for some reason we need to cancel. As a result, the execution can then continue from a consistent state as if that suppressed action had not been executed at all. In particular, these mechanisms allow for the correct implementation of transactions $[7,8]$, that are partial computations which either are totally executed or they are not executed at all. This includes the modification of information in data bases, so that we never include an 'incomplete' set of related updates that would produce an inconsistent state. In such a state one could infer some pieces of information that do not match, due to the fact that the modification procedure has not been satisfactorily completed. Another typical example would be the transactions between financial institutions, 
for instance, when transferring money, or nowadays any e-commerce platform, where the payments received should match the distributed goods [6].

Within the domain of Formal Methods, reversibility has been studied, for instance, in the framework of process calculi [17, 15], event structures [18], DNAcomputing [5], category theory [9], as well as within the field of quantum computing [20]. In the latter case, reversibility plays a central role due to the inherent reversibility of the mechanisms on which quantum computing is based. On the other hand, in Petri nets reversibility is usually understood as a global property. Historically it was considered in a sense closer to its meaning in process calculi [13], but such a local reversibility within the framework of Petri Nets has not been yet extensively studied. This is quite surprising as the formalization of transitions by means of pairs of precondition and postcondition places gives one an immediate way of defining the reversal of a transition simply by interchanging those two sets. There are, however, some more recent approaches that either focus on the structural study of Petri Nets [14], or on their algebraic study by means of invariants [16].

The approach presented in this paper is more operational, and extends the study of reversing (sequential) transitions initiated in [4], where it was shown that the apparent simplicity of this approach is far from trivial, mainly due to the difficulty of avoiding a situation that the added reversing transitions are fired in an inconsistent way; for instance, before the transition to be reversed was fired at all. [3] continued the study considering the particular case of bounded Petri nets, and distinguishing between strict reverses and effect reverses. The latter produce the effect of reversing the original transitions, but possibly with increasing or reducing the conditions checked for the reversed firing. It was shown that some transition systems which can be solved by a bounded net allow the reversal of their transitions by means of single reversing transitions, while in some other cases the reversal is only possible if we allow the splitting of reverses. This means that one can have a collection of reverses for the same transition, and each of them will be only fired at some of the markings, where the reversal of the original transition must be possible.

In [3] only the sequential (interleaving) semantics of nets was considered and, in fact, several of the presented examples were just (finite) trace systems, taking advantage of the results presented in [2,12], where binary words representable by Petri net were characterised. The latter problem and its consequences for reversibility has been recently further investigated in [11].

In this paper, we initiate the study of step reversing assuming the step semantics of Petri nets. We assume that the transition systems to be synthesized include the information about the multisets of enabled transitions that should be fireable in parallel. The reversal of the transitions should preserve this step information so that the simultaneous firing of several reverse transitions should exactly correspond to the original steps at the system represented by a Petri net.

Using Petri nets as a system model, we introduce concepts related to this new scenario, generalising notions used in the single action case. Since our aim now is to reverse steps, the simple definition which worked in the sequential case is 
no longer sufficient. When looking for the adequate generalization defining step reversing, we have found that two (non equivalent) definitions look 'natural'. The former only allows steps which comprise either the original actions, or the reverse actions (direct reversibility). The latter allows also mixing of these two kinds of actions (mixed reversibility). It turns out that these two ways of interpreting reversibility of steps cause very big differences. Crucially, it appears that the direct reversibility cannot be implemented for steps which are true multisets, and so in such a case one has to aim at mixed reversibility. In this way we have found a striking difference between reversing steps which are sets and those which are true multisets (when autoconcurrency of actions in system executions is allowed). However, we still have a general positive result which shows that whenever sequential reversing is possible, once the steps of the system have been satisfactorily represented, we obtain also a sound reversal of those steps.

The paper is organised as follows. In the next section we recall a number of notions and notations used throughout the paper. We also introduce the direct and mixed step reversibility. In Section 4, we show that the direct reversibility cannot be achieved in the presence of autoconcurrency. The following section presents our positive results about lifting of sequential reversibility to step reversibility, by taking into account autoconcurrency. In Section 6, we develop results which show that in many cases the reversibility problem can be reduced to the net synthesis problem. The paper ends with some concluding remarks.

\section{Preliminaries}

Multisets. A multiset over a finite set $X$ is a mapping $\alpha: X \rightarrow \mathbb{N}$, where $\mathbb{N}$ is the set of non-negative integers. The set of all multisets over $X$ is denoted by $\operatorname{mult}(X) . \alpha+\beta$ and $\alpha-\beta$ denote the multiset sum and difference, i.e., $(\alpha+\beta)(x)=\alpha(x)+\beta(x)$ and $(\alpha-\beta)(x)=\alpha(x)-\beta(x)$, for every $x \in X$. Note that $\alpha-\beta$ is defined provided that $\beta \leq \alpha$ which means that $\beta(x) \leq \alpha(x)$, for every $x \in X$. The size of $\alpha$ is defined as $|\alpha|=\sum_{x \in X} \alpha(x)$, and the support as the set $\operatorname{supp}(\alpha)=\{x \in X \mid \alpha(x) \geq 1\}$. We also denote $x \in \alpha$ if $\alpha(x) \geq 1$. For $Y \subseteq X, \alpha \cap Y$ denotes multiset $\beta$ (still over $X$ ) such that $\beta(y)=\alpha(y)$, for $y \in Y$, and $\beta(x)=0$, for $x \in X \backslash Y$. Subsets of $X$ can be identified with multisets which return values in $\{0,1\}$, and its elements with singleton sets (i.e., multisets of size one). The empty (multi)set is denoted by $\varnothing$; a multiset $\alpha$ such that $\alpha(a)=2$, $\alpha(b)=1$, and $\alpha(X \backslash\{a, b\})=\{0\}$, can be denoted by $(a a b)$; and $a^{k}$ denotes multiset $\alpha$ such that $\alpha(a)=k$ and $\alpha(X \backslash\{a\})=\{0\}$.

Step transition systems. A step transition system is defined as a tuple STS $=$ $\left(S, T, \rightarrow, s_{0}\right)$ such that $S$ is a nonempty set of states, $T$ is a finite set of actions, $\rightarrow \subseteq S \times \operatorname{mult}(T) \times S$ is the set of arcs (also called transitions), and $s_{0} \in S$ is the initial state. The labels in mult $(T)$ represent simultaneous executions of groups of actions, called steps. Rather than $(s, \alpha, r) \in \rightarrow$, we can denote $s \stackrel{\alpha}{\longrightarrow}$ STS $r$ or $s \stackrel{\alpha}{\longrightarrow} r$ or $r \stackrel{\alpha}{\longleftarrow} s$. Moreover, $s \stackrel{\alpha}{\longrightarrow}$ STS or $s \stackrel{\alpha}{\longrightarrow}$ means that there is some $r$ such that $s \stackrel{\alpha}{\longrightarrow} S T S$. 
A state $r$ is reachable from state $s$ if there are steps $\alpha_{1}, \ldots, \alpha_{k}(k \geq 0)$ and states $s_{1}, \ldots, s_{k+1}$ such that $s=s_{1} \stackrel{\alpha_{1}}{\longrightarrow} s_{2} \ldots s_{k} \stackrel{\alpha_{k}}{\longrightarrow} s_{k+1}=r$. We denote this by $s \stackrel{\alpha_{1} \ldots \alpha_{k}}{\longrightarrow} S T S$ or $s \stackrel{\alpha_{1} \ldots \alpha_{k}}{\longrightarrow} r$ or $r \stackrel{\alpha_{k} \ldots \alpha_{1}}{\longleftarrow} s$. The set of all states from which a state $s$ is reachable is denoted by $\operatorname{pred}(s)$, and $s$ is a home state if $\operatorname{pred}(s)=S$. Moreover, a set of states $S^{\prime} \subseteq S$ is a home cover of $S T S$ if $S=\bigcup_{s \in S^{\prime}} \operatorname{pred}(s)$.

STS is state-finite if $S$ is finite, step-finite if $\left\{\alpha \mid s \stackrel{\alpha}{\longrightarrow} s^{\prime}\right\}$ is finite, and finite if it is both state- and step-finite (and so $\rightarrow$ is also finite).

Step transition systems are intended here to capture (step) reachability graphs of Petri nets. Of course, not every step transition system can be such a graph. To reflect this, we formulate first the following properties of STS:

FD forward deterministism

$s^{\prime} \stackrel{\alpha}{\longleftarrow} s \stackrel{\alpha}{\longrightarrow} s^{\prime \prime}$ implies $s^{\prime}=s^{\prime \prime}$. Then we can define $s \oplus \alpha=s^{\prime}$.

$\boldsymbol{B D} \quad$ backward deterministism

$s^{\prime} \stackrel{\alpha}{\longrightarrow} s \stackrel{\alpha}{\longleftarrow} s^{\prime \prime}$ implies $s^{\prime}=s^{\prime \prime}$. Then we can define $s \ominus \alpha=s^{\prime}$.

$\boldsymbol{R} \boldsymbol{E} \boldsymbol{A}$ reachability

$s_{0} \in \operatorname{pred}(s)$, for every $s \in S$.

SEQ $Q$ sequentialisability

$s \stackrel{\alpha}{\longrightarrow}$ implies $s \stackrel{\alpha_{1} \ldots \alpha_{k}}{\longrightarrow}$, whenever $\alpha=\sum_{i=1}^{k} \alpha_{i}$.

$\boldsymbol{E L}$ empty loops

$s \stackrel{\varnothing}{\longrightarrow} s$, for every $s \in S .^{4}$

FC forward confluence

$s^{\prime} \stackrel{\alpha_{k} \ldots \alpha_{1}}{\longleftarrow} s \stackrel{\beta_{1} \ldots \beta_{m}}{\longrightarrow} s^{\prime \prime}$ implies $s^{\prime}=s^{\prime \prime}$, whenever $\sum_{i=1}^{k} \alpha_{i}=\sum_{j=1}^{m} \beta_{j}$.

BC backward confluence

$s^{\prime} \stackrel{\alpha_{1} \ldots \alpha_{k}}{\longrightarrow} s \stackrel{\beta_{m} \ldots \beta_{1}}{\longleftarrow} s^{\prime \prime}$ implies $s^{\prime}=s^{\prime \prime}$, whenever $\sum_{i=1}^{k} \alpha_{i}=\sum_{j=1}^{m} \beta_{j}$.

Any STS satisfying the above properties will be called, in this paper, a wellformed step transition system (or WFST-System). Note that $F C$ and $B C$ respectively generalise $F D$ and $B D$, and $E L \mathscr{E} F D$ means that $s \stackrel{\varnothing}{\longrightarrow} s^{\prime} \Longleftrightarrow s=s^{\prime}$.

Proposition 1. Let $S T S=\left(S, T, \rightarrow, s_{0}\right)$ be a WFST-system and $s \in S$. If $s \oplus \alpha$ is defined and $\beta+\gamma \leq \alpha$, then $s \oplus \beta, s \oplus(\beta+\gamma)$ and $(s \oplus \beta) \oplus \gamma$ are also defined, and $(s \oplus \beta) \oplus \gamma=s \oplus(\beta+\gamma)$.

Proof. By $s \stackrel{\alpha}{\longrightarrow}$ and SEQ $\&$ FD, we have $s \stackrel{\beta}{\longrightarrow} s \oplus \beta \stackrel{\gamma}{\longrightarrow}(s \oplus \beta) \oplus \gamma$ as well as $s \stackrel{\beta+\gamma}{\longrightarrow} s \oplus(\beta+\gamma)$. Hence, by $F C,(s \oplus \beta) \oplus \gamma=s \oplus(\beta+\gamma)$.

Being well-formed does not still characterise step transition systems defined by PT-nets. A complete characterisation can be obtained using, e.g., theory of regions $[1,10]$. However, we will not need here such a characterisation, since we are only interested in obtaining sufficient conditions for the representability of

\footnotetext{
${ }^{4}$ Arcs labelled with the empty multiset will not be usually depicted.
} 
step transition systems by PT-nets, starting from the existing results about the representability of ordinary (sequential) transition systems.

Let $S T S=\left(S, T, \rightarrow, s_{0}\right)$ and $S T S^{\prime}=\left(S^{\prime}, T^{\prime}, \rightarrow^{\prime}, s_{0}^{\prime}\right)$ be step transition systems. Then $S T S$ is:

- a sub-system of $S T S^{\prime}$ if $S \subseteq S^{\prime}, T \subseteq T^{\prime}, \rightarrow \subseteq \rightarrow^{\prime}$, and $s_{0}=s_{0}^{\prime}$. We denote this by $S T S \hookrightarrow S T S^{\prime}$.

- included in $S T S^{\prime}$, if $T \subseteq T^{\prime}$, and there is a bijection $\psi$ with the domain containing $S$ such that $\left\{\left(\psi(s), \alpha, \psi\left(s^{\prime}\right)\right) \mid s \stackrel{\alpha}{\longrightarrow} s^{\prime}\right\} \subseteq \rightarrow^{\prime}, \psi(S)=S^{\prime}$, and $\psi\left(s_{0}\right)=s_{0}^{\prime}$. We denote this by $S T S \triangleleft_{\psi} S T S^{\prime}$ or $S T S \triangleleft S T S^{\prime}$.

- isomorphic with $S T S^{\prime}$ if $S T S \triangleleft_{\psi} S T S^{\prime}$ and $S T S^{\prime} \triangleleft_{\psi^{-1}} S T S$, for some $\psi \cdot{ }^{5}$ We denote this by $S T S \simeq_{\psi} S T S^{\prime}$ or $S T S \simeq S T S^{\prime}$.

We also define three ways of removing transitions from a step transition system:

$$
\begin{aligned}
& S T S^{s e q}=\left(S, T,\{(s, \alpha, r)|s \stackrel{\alpha}{\longrightarrow} r \wedge| \alpha \mid \leq 1\}, \quad s_{0}\right) \\
& S T S^{\text {set }}=\left(S, T,\{(s, \alpha, r) \mid s \stackrel{\alpha}{\longrightarrow} r \wedge \operatorname{supp}(\alpha)=\alpha\}, s_{0}\right) \\
& S T S^{\text {spike }}=\left(S, T,\{(s, \alpha, r)|s \stackrel{\alpha}{\longrightarrow} r \wedge| \operatorname{supp}(\alpha) \mid \leq 1\}, s_{0}\right) \text {. }
\end{aligned}
$$

That is, $S T S^{s e q}$ is obtained by only retaining singleton steps and $\varnothing, S T S^{s e t}$ by only retaining steps which are sets, and $S T S^{\text {spike }}$ by removing all steps which use more than one action. Then STS is a sequential / set / spiking step transition system if respectively $S T S=S T S^{\text {seq }} / S T S=S T S^{\text {set }} / S T S=S T S^{\text {spike }}{ }^{6}$

For a step transition system $S=\left(S, T, \rightarrow, s_{0}\right)$ and $T^{\prime} \subseteq T$, the subsystem of $\mathrm{S}$ induced by $T^{\prime}$ is $\left.S T S\right|_{T^{\prime}}=\left(S, T^{\prime},\left\{\left(s, \alpha, s^{\prime}\right) \mid s \stackrel{\alpha}{\longrightarrow} s^{\prime} \wedge \alpha \in \operatorname{mult}\left(T^{\prime}\right)\right\}, s_{0}\right) .{ }^{7}$

Place/Transion-nets. A Place/Transition net (or PT-net) [19] is a tuple $N=\left(P, T, F, M_{0}\right)$, where $P$ is a finite set of places, $T$ is a disjoint finite set of transitions (or actions), $F$ is the flow function $F:((P \times T) \cup(T \times P)) \rightarrow \mathbb{N}$ specifying the arc weights, and $M_{0}$ is the initial marking (where a marking - a global state - is a multiset over $P)$. Moreover, $(P, T, F)$ is an unmarked PT-net.

Multisets over $T$ - called again steps - represent executions of groups of transitions. The effect of a step $\alpha$ is a multiset of places eff $_{N}(\alpha)=$ post $_{N}(\alpha)-$ $\operatorname{pre}_{N}(\alpha)$, where, for every $p \in P$ :

$$
\operatorname{pre}_{N}(\alpha)(p)=\sum_{t \in T} \alpha(t) \cdot F(p, t) \text { and } \operatorname{post}_{N}(\alpha)(p)=\sum_{t \in T} \alpha(t) \cdot F(t, p) .
$$

A step $\alpha$ is enabled at a marking $M$ if $M \geq \operatorname{pre}_{N}(\alpha)$. We denote this by $M[\alpha\rangle_{N}$. The firing of such a step $M$ leads to marking $M^{\prime}=M+e f f_{N}(\alpha)$. We denote this by $M[\alpha\rangle_{N} M^{\prime}$. Note that $M[\alpha\rangle_{N}$ implies $M[\beta\rangle_{N}$, for every $\beta \leq \alpha$. Moreover, $M[\alpha+\beta\rangle_{N}$ implies: $M[\alpha\rangle_{N} M+e^{e f f_{N}}(\alpha)[\beta\rangle_{N} M+e f f f_{N}(\alpha+\beta)$. The set reach

\footnotetext{
${ }^{5}$ If $S T S$ and $S T S^{\prime}$ are well-formed, then $\psi$ is unique due to FD $\& R E A$.

${ }^{6}$ If $S T S$ is well-formed, then $S T S^{s e q}, S T S^{\text {set }}$, and $S T S^{\text {spike }}$ satisfy $R E A$ due to $R E A \& S E Q$.

${ }^{7}$ Note that $\left.S T S\right|_{T^{\prime}}$ may be not $R E A$ even for $S T S$ that is $R E A$.
} 
of reachable markings is the smallest set of markings such that $M_{0} \in$ reach $_{N}$ and if $M \in$ reach $_{N}$ and $M[\alpha\rangle_{N} M^{\prime}$, for some $\alpha$, then $M^{\prime} \in$ reach $_{N}$. The overall behaviour of $N$ can be captured by its concurrent reachability graph defined as $C R G_{N}=\left(\right.$ reach $_{N}, T,\left\{\left(M, \alpha, M^{\prime}\right) \mid M \in\right.$ reach $\left.\left._{N} \wedge M[\alpha\rangle_{N} M^{\prime}\right\}, M_{0}\right) . C R G_{N}$ is a WFST-system, and $M \stackrel{\alpha}{\longrightarrow} M_{N} M^{\prime}$ will denote that $M \stackrel{\alpha}{\longrightarrow} C_{R G_{N}} M^{\prime}$.

A step transition system $S T S$ is solvable if there is a PT-net $N$ such that $S T S \simeq C R G_{N}$. Moreover, step transition systems $S T S_{r}=\left(S_{r}, T, \rightarrow_{r}, s_{r}\right)$ (for $r \in R$ ) are simultaneously solvable if there are PT-nets $N_{r}=\left(P, T, F, M_{r}\right)$ (for $r \in$ $R$ ) and a bijection $\psi: \bigcup_{r \in R} S_{r} \rightarrow \bigcup_{r \in R}$ reach $_{N_{r}}$ such that $S T S_{r} \simeq_{\psi} C R G_{N_{r}}$, for every $r \in R$. (Note that the $S_{r}$ 's need not be disjoint.)

For a PT-net $N=\left(P, T, F, M_{0}\right)$ and $T^{\prime} \subseteq T$, the PT-(sub)net of $\mathrm{N}$ induced by $T^{\prime}$ is $\left.N\right|_{T^{\prime}}=\left(P, T^{\prime}, F_{\left(P \times T^{\prime}\right) \cup\left(T^{\prime} \times P\right)}, M_{0}\right)$.

\section{$3 \quad$ Reversing steps}

A reverse of an action or net transition $x$ will be denoted by $\bar{x}$, and for a multiset $X=\left(x_{1} \ldots x_{k}\right)$ with $k \geq 0$, we denote $\bar{X}=\left(\bar{x}_{1} \ldots \bar{x}_{k}\right)$.

Reversing in transition systems. We introduce three ways in which one can modify a step transition system in order to capture the effect of reversing actions.

The direct/set/mixed reverse of a step transition system $S T S=(S, T, \rightarrow$ ,$\left.s_{0}\right)$ satisfying $S E Q \& F D$ is respectively given by:

$$
\begin{aligned}
& S T S^{\text {rev }}=\left(S, T \cup \bar{T}, \rightarrow \cup \rightarrow_{\text {rev }}, s_{0}\right) \\
& S T S^{\text {srev }}=\left(S, T \cup \bar{T}, \rightarrow \cup \rightarrow_{\text {srev }}, s_{0}\right) \\
& S T S^{\text {mrev }}=\left(S, T \cup \bar{T}, \rightarrow_{\text {mrev }}, \quad s_{0}\right), \text { where: } \\
& \rightarrow_{\text {rev }}=\{(s \oplus \alpha, \bar{\alpha}, s) \mid s \stackrel{\alpha}{\longrightarrow}\} \\
& \rightarrow_{\text {srev }}=\{(s \oplus \alpha, \bar{\alpha}, s) \mid s \stackrel{\alpha}{\longrightarrow} \wedge \operatorname{supp}(\alpha)=\alpha\} \\
& \rightarrow_{\text {mrev }}=\{(s \oplus \alpha, \bar{\alpha}+\beta, s \oplus \beta) \mid s \stackrel{\alpha+\beta}{\longrightarrow}\} .
\end{aligned}
$$

Therefore, $\rightarrow_{\text {rev }}$ reverses all the (original) steps: $\rightarrow_{\text {srev }}$ only reverses the steps that are sets; and finally $\rightarrow_{\text {mrev }}$ introduces partial reverses, which means mixed steps, including both original and reversed actions.

Figure 1 illustrates the idea of mixed reversing. Note that $s \oplus \alpha$ and $s \oplus \beta$ above are well-defined states in $S T S$ due to $S E Q \& F D$.

Proposition 2. Let STS be a WFST-system, and $\alpha, \beta$ be steps of its actions.

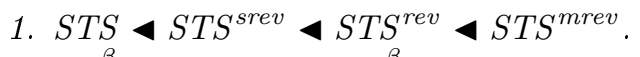

2. $s \stackrel{\beta}{\longrightarrow}$ STS mrev $s^{\prime}$ iff $s \stackrel{\beta}{\longrightarrow}$ STS $s^{\prime}$.

3. $s \stackrel{\bar{\alpha}}{\longrightarrow}$ STS mrev $s^{\prime}$ iff $s \stackrel{\bar{\alpha}}{\longrightarrow}$ STS rev $s^{\prime}$.

4. $s \stackrel{\alpha+\beta}{\longrightarrow}$ STS implies $s \oplus \alpha \stackrel{\bar{\delta}+\gamma}{\longrightarrow}$ STSmrev $s \oplus(\gamma+\alpha-\delta)$,

for all $\delta \leq \alpha$ and $\gamma \leq \beta$. 


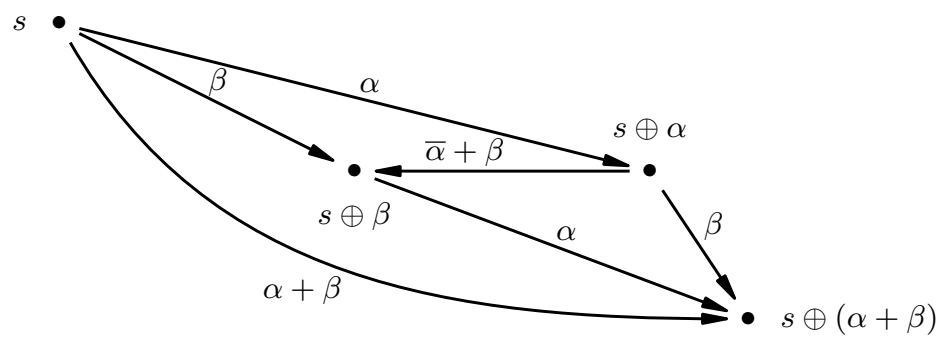

Fig. 1. A mixed reverse transition $s \oplus \alpha \stackrel{\bar{\alpha}+\beta}{\longrightarrow}$ mrev $s \oplus \beta$ derived from $s \stackrel{\alpha+\beta}{\longrightarrow}$.

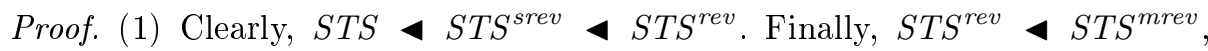
because we can take $\alpha=\varnothing$ and then $s \oplus \alpha=s$ using that $S T S$ satisfies FD $\& E L$; and also $\rightarrow_{\text {rev }} \subseteq \rightarrow_{\text {mrev }}$ can be obtained in a similar way, taking $\beta=\varnothing$.

(2) By part (1), we only need to show that $s \stackrel{\beta}{\longrightarrow}$ STSmrev $s^{\prime}$ implies $s \stackrel{\beta}{\longrightarrow}$ STS $s^{\prime}$. Indeed, given that $\beta=\bar{\varnothing}+\beta$ and $S T S$ satisfies $F D$, the former implies that there is $r \in S$ such that $r \stackrel{\varnothing+\beta}{\longrightarrow} S T S r \oplus \beta, s=r \oplus \varnothing$, and $s^{\prime}=r \oplus \beta$. Hence, by $E L \mathscr{E} B D$ for $S T S, s=r$. Thus $s \stackrel{\beta}{\longrightarrow} S T S s^{\prime}$.

(3) By part (1), we only need to show that $s \stackrel{\bar{\alpha}}{\longrightarrow}$ STS mrev $s^{\prime}$ implies $s \stackrel{\bar{\alpha}}{\longrightarrow}$ STS rev $s^{\prime}$. Indeed, given that $\bar{\alpha}=\bar{\alpha}+\varnothing$ and $S T S$ satisfies $F D$, the former implies that there is $r \in S$ such that $r \stackrel{\alpha+\varnothing}{\longrightarrow} S T S r \oplus \alpha, s=r \oplus \alpha$, and $s^{\prime}=r \oplus \varnothing$. Hence, by $E L \mathscr{E} B D$ for $S T S, s^{\prime}=r$. Thus $s \stackrel{\alpha}{\longrightarrow}$ STS $s^{\prime}$ so $s^{\prime} \stackrel{\bar{\alpha}}{\longrightarrow}$ STS rev $s$.

(4) By $s \stackrel{\alpha+\beta}{\longrightarrow} S T S$ and $S E Q \mathscr{G} F D$ for $S T S, s \stackrel{\alpha-\delta}{\longrightarrow} S T S \cdot \varphi(\alpha-\delta) \stackrel{\delta+\gamma}{\longrightarrow} S T S$. Hence, by the definition of $S T S^{m r e v}$,

$$
(s \oplus(\alpha-\delta)) \oplus \delta \stackrel{\bar{\delta}+\gamma}{\longrightarrow}{ }_{S T S^{\text {mrev }}}(s \oplus(\alpha-\delta)) \oplus \gamma .
$$

Moreover, by $s \stackrel{\alpha+\beta}{\longrightarrow}$ STS and Proposition 1,

$$
\begin{aligned}
& (s \oplus(\alpha-\delta)) \oplus \delta=s \oplus((\alpha-\delta)+\delta)=s \oplus \alpha \\
& (s \oplus(\alpha-\delta)) \oplus \gamma=s \oplus((\alpha-\delta)+\gamma)=s \oplus(\gamma+\alpha-\delta) .
\end{aligned}
$$

In general, $S T S^{r e v}, S T S^{s r e v}$, and $S T S^{\text {mrev }}$ need not be well-formed even though $S T S$ was. However, the only properties which may fail to carry over from $S T S$ are the two versions of confluence.

Example 1. The step transition system in Figure 2(a) is well-formed. However, adding reversals destroys forward confluence, as demonstrated in Figure 2(b). Reversing the arcs results in a symmetric counterexample for the preservation of backward confluence.

Proposition 3. If STS satisfies FD \& BD E REA \&SEQ E EL, then the step transition systems $S T S^{m r e v}, S T S^{\text {srev }}$, and $S T S^{r e v}$ also satisfy them. 
(a)

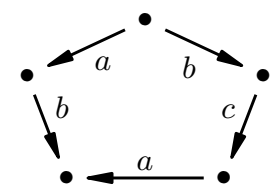

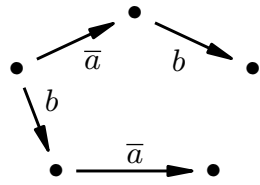

(b)

Fig. 2. Reversing does not preserve confluence.

Proof. By Proposition 2(1), the result follows immediately for $E L$ and $R E A$. For the remaining three properties, by Proposition $2(2,3)$, it suffices to show it for $S T S^{\text {mrev }}$. To this end, suppose that:

$$
\begin{array}{ll}
s \stackrel{\alpha+\beta}{\longrightarrow} \text { STS } & s \oplus \alpha \stackrel{\bar{\alpha}+\beta}{\longrightarrow} S_{S T S^{\text {mrev }}} s \oplus \beta \\
s^{\prime} \stackrel{\alpha+\beta}{\longrightarrow} \text { STS } & s^{\prime} \oplus \alpha \stackrel{\bar{\alpha}+\beta}{\longrightarrow} S_{S^{\text {mrev }}} s^{\prime} \oplus \beta .
\end{array}
$$

Then, by $S E Q \& F D$ for $S T S$, we have:

$$
\begin{array}{ll}
s \stackrel{\alpha}{\longrightarrow} S T S=\alpha & s \stackrel{\beta}{\longrightarrow}{ }_{S T S} s \oplus \beta \\
s^{\prime} \stackrel{\alpha}{\longrightarrow} S T S s^{\prime} \oplus \alpha & s^{\prime} \stackrel{\beta}{\longrightarrow} S_{S S} s^{\prime} \oplus \beta .
\end{array}
$$

Suppose now that $s \oplus \alpha=s^{\prime} \oplus \alpha$. Then, by $B D$ for $S T S, s=s^{\prime}$. Hence, by $F D$ for $S T S, s \oplus \beta=s^{\prime} \oplus \beta$. As a result, $F D$ holds for $S T S^{m r e v}$. The proof of $B D$ is symmetric.

To prove $S E Q$ for $S T S^{\text {mrev }}$, it suffices to consider $k=2$. Suppose that:

$$
s \stackrel{\alpha_{1}+\alpha_{2}+\beta_{1}+\beta_{2}}{\longrightarrow} S T S \text { and } s \oplus\left(\alpha_{1}+\alpha_{2}\right) \stackrel{\bar{\alpha}_{1}+\bar{\alpha}_{2}+\beta_{1}+\beta_{2}}{\longrightarrow}{ }_{S T S^{\text {mrev }}} s \oplus\left(\beta_{1}+\beta_{2}\right) \text {. }
$$

Then, by $S E Q$ for $S T S$, we have $s \oplus \alpha_{2}{\stackrel{\alpha_{1}+\beta_{1}}{\longrightarrow}}_{S T S}$ and $s \oplus \beta_{1}{\stackrel{\alpha_{2}+\beta_{2}}{\longrightarrow}}_{S T S}$. Hence, by the definition of $S T S^{\text {mrev }}$,

$$
\begin{aligned}
& \left(s \oplus \alpha_{2}\right) \oplus \alpha_{1} \stackrel{\bar{\alpha}_{1}+\beta_{1}}{\longrightarrow}{ }_{\text {STS mrev }}\left(s \oplus \alpha_{2}\right) \oplus \beta_{1} \\
& \left(s \oplus \beta_{1}\right) \oplus \alpha_{2} \stackrel{\bar{\alpha}_{2}+\beta_{2}}{\longrightarrow} \text { STS } S^{\text {mrev }}\left(s \oplus \beta_{1}\right) \oplus \beta_{2} .
\end{aligned}
$$

Moreover, by Proposition 1, we have:

$$
\begin{aligned}
& s \oplus\left(\alpha_{2}+\alpha_{1}\right)=\left(s \oplus \alpha_{2}\right) \oplus \alpha_{1} \\
&\left(s \oplus \alpha_{2}\right) \oplus \beta_{1}=s \oplus\left(\alpha_{2}+\beta_{1}\right)=\left(s \oplus \beta_{1}\right) \oplus \beta_{2}=s \oplus\left(\beta_{1}+\beta_{2}\right) \\
&\left(s \oplus \beta_{1}\right) \oplus \alpha_{2} .
\end{aligned}
$$

Hence, we obtain:

$$
s \oplus\left(\alpha_{1}+\alpha_{2}\right) \stackrel{\bar{\alpha}_{1}+\beta_{1}}{\longrightarrow} \text { STS mrev } s \oplus\left(\alpha_{2}+\beta_{1}\right) \stackrel{\bar{\alpha}_{2}+\beta_{2}}{\longrightarrow} \text { STSmrev } s \oplus\left(\beta_{1}+\beta_{2}\right),
$$

which means that $S E Q$ holds for $S T S^{m r e v}$.

Proposition 4. $s \stackrel{\bar{\alpha}+\beta}{\text { STS mrev }} s^{\prime}$ iff $s^{\prime} \stackrel{\alpha+\bar{\beta}}{\longrightarrow}$ STSmrev $s$, for every WFST-system STS. 
Proof. Since both implications really state the same, it suffices to show any of them. Suppose that $s \stackrel{\bar{\alpha}+\beta}{\longrightarrow}$ STS $S^{\text {mrev }} s^{\prime}$. Then there is $r$ such that $r \stackrel{\alpha+\beta}{\longrightarrow}$ STS $s=r \oplus \alpha$, and $s^{\prime}=r \oplus \beta$ and then we only need to swap the roles of $\alpha$ and $\beta$ to conclude $r \oplus \beta \stackrel{\alpha+\bar{\beta}}{\longrightarrow}$ STS mrev $r \oplus \alpha$.

Reversing in nets. Due to the natural decomposability character of steps made up of net transitions, adding reverses to PT-nets is done at the level of transitions rather than steps:

- A PT-net $N$ with reverses is such that, for each original transition $t$, there is a reverse transition $\bar{t}$ with the opposite effect, i.e., eff ${ }_{N}(t)=-e f f_{N}(\bar{t})$.

- A PT-net $N$ with strict reverses is such that, for each original transition $t$, there is a reverse transition $\bar{t}$ with the opposite connectivity, i.e., $\operatorname{pre}_{N}(\bar{t})=$ $\operatorname{post}_{N}(t)$ and $\operatorname{post}_{N}(\bar{t})=\operatorname{pre}_{N}(t)$.

Proposition 5. If STS is a solvable step transition system, then STS ${ }^{\text {rev }}$ and STS ${ }^{\text {mrev }}$ are WFST-systems.

Proof. Since $S T S=\left(S, T, \rightarrow, s_{0}\right)$ is solvable, there is a PT-net $N=\left(P, T, F, M_{0}\right)$ and a bijection $\psi: S \rightarrow$ reach $_{N}$ such that $S T S \simeq_{\psi} C R G_{N}$. Hence, since $C R G_{N}$ is well-formed, STS is also well-formed. Below $M_{s}=\psi(s)$, for every $s \in S$.

It suffices to show that $S T S^{\text {mrev }}$ is well-formed, and, by Proposition 3, we only need to check that $F C$ and $B C$ hold for $S T S^{m r e v}$. Suppose that: $s \stackrel{\alpha+\beta}{\longrightarrow} S T S$ and $s \oplus \alpha \stackrel{\bar{\alpha}+\beta}{\longrightarrow} S T S^{\text {mrev }} s \oplus \beta$. Then, by $F D \& B D$ for $C R G_{N}$, we have:

$$
M_{s \oplus \beta}=M_{s}+e f f_{N}(\beta)=M_{s \oplus \alpha}-e_{f f}(\alpha)+e f f_{N}(\beta) .
$$

Therefore, if $s \stackrel{\left(\bar{\alpha}_{1}+\beta_{1}\right) \ldots\left(\bar{\alpha}_{k}+\beta_{k}\right)}{\longrightarrow}$ STS mrev $s^{\prime}$, then:

$$
M_{s^{\prime}}=M_{s}-\sum_{i=1}^{k} e_{N}\left(\alpha_{i}\right)+\sum_{i=1}^{k} \operatorname{eff}_{N}\left(\beta_{i}\right) .
$$

Hence both $F C$ and $B C$ hold for $S T S^{m r e v}$.

As an immediate consequence, we obtain the following a characterisation.

Corollary 1. If STS is a WFST-system, but STS ${ }^{\text {mrev }}$ is not, then STS is not solvable.

\section{Multisets and mixed reversibility}

Our investigation of step reversibility starts with a straightforward but pivotal result stating that, in the domain of PT-nets, direct reversibility cannot handle steps which are true multisets.

Proposition 6. Let STS be a WFST-system which is not a set transition system. Then STS ${ }^{\text {rev }}$ is not solvable. 
Proof (See Figure 3(a)). Let $S T S=\left(S, T, \rightarrow, s_{0}\right)$ and $N=\left(P, T \cup \bar{T}, F, M_{0}\right)$ be a PT-net such that $S T S^{r e v} \simeq_{\psi} C R G_{N}$.

Suppose that $v \stackrel{\alpha}{\longrightarrow}$ and $(a a) \leq \alpha$. Then, since $S T S$ satisfies $S E Q$, there are $w, q \in S$ such that $v \stackrel{(a a)}{\longrightarrow} w$ and $v \stackrel{a}{\longrightarrow} q$.

Let $M_{x}=\psi(x)$, for $x \in\{v, w, q\}$. By $S T S^{r e v} \simeq_{\psi} C R G_{N}$, the step $(a \bar{a})$ is not enabled at $M_{q}$. Hence, there must be $p \in P$ such that

$$
M_{q}(p)<F(p, \bar{a})+F(p, a) .
$$

On the other hand, $(a a)$ is enabled at $M_{v}$, and $(\overline{a a})$ is enabled at $M_{w}$. Hence $M_{v}(p) \geq 2 \cdot F(p, a)$ and $M_{w}(p) \geq 2 \cdot F(p, \bar{a})$. We also have:

$$
\begin{aligned}
& M_{w}(p)=M_{v}(p)+2 \cdot F(a, p)-2 \cdot F(p, a) \\
& M_{q}(p)=M_{v}(p)+F(a, p)-F(p, a) .
\end{aligned}
$$

Thus we obtain:

$$
\begin{aligned}
2 \cdot F(p, \bar{a})+2 \cdot F(p, a) & \leq M_{v}(p)+M_{w}(p) \\
& =2 \cdot M_{v}(p)+2 \cdot F(a, p)-2 \cdot F(p, a),
\end{aligned}
$$

and so $F(p, \bar{a})+F(p, a) \leq M_{v}(p)+F(a, p)-F(p, a)=M_{q}(p)$, yielding a contradiction with $(1)$.

(a)
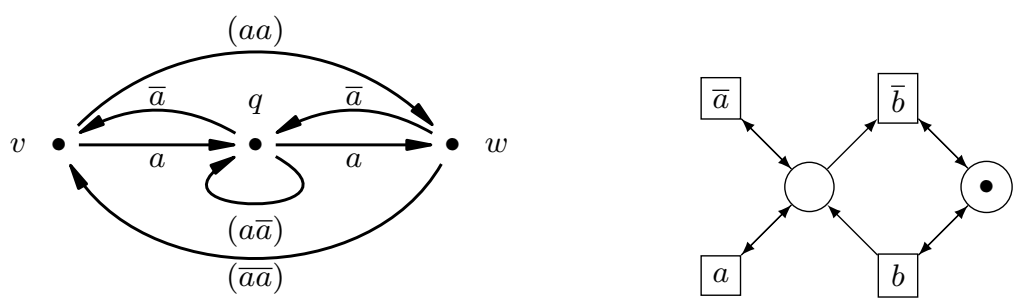

(b)

Fig. 3. An illustration of the proof of Proposition $6(a)$, and PT-net generating concurrent reachability graph which is not step-finite $(b)$.

A result similar to Proposition 6 does not hold for $S T S^{m r e v}$ since, in this case it may contain, in particular, the mixed step $(a \bar{a})$ that was needed in the proof of the last result (a suitable counterexample can be provided by a WFST-system which 'executes' the diamond of $(a a)$ ). Hence, in the case of step (but not set) transition systems, it makes sense to investigate mixed reversibility, rather than direct reversibility, which we have proved to be impossible.

Proposition 7. Let STS be a step-finite WFST-system. If $S T S^{m r e v}$ is solvable, then $S T S^{\text {srev }}$ is also solvable.

Proof. Since $S T S$ is step-finite, there is $k \geq 1$ such that $|\alpha| \leq k$, whenever $s \stackrel{\alpha}{\longrightarrow} S T S$. Moreover, since $S T S^{m r e v}$ is solvable, there exists a PT-net $N=(P, T \cup$ $\left.\bar{T}, F, M_{0}\right)$ such that $S T S^{m r e v} \simeq_{\psi} C R G_{N}$. We then modify $N$, getting a new net 
$N^{\prime}$, by adding to $P$ a set of fresh places $P^{\prime}=\left\{p_{t u} \mid t \in T \wedge u \in \bar{T}\right\}$. Each $p_{t u}$ is such that $M_{0}\left(p_{t u}\right)=k$ and has four non-zero connections:

$$
F\left(t, p_{t u}\right)=F\left(p_{t u}, t\right)=1 \text { and } F\left(u, p_{t u}\right)=F\left(p_{t u}, u\right)=k .
$$

For the obtained PT-net $N^{\prime}, S T S^{\text {srev }} \simeq_{\psi^{\prime}} C R G_{N^{\prime}}$ where, for every state $s$ of $S T S, \psi^{\prime}(s)=\psi(s)+\sum_{p \in P^{\prime}} p^{k}$.

Example 2. The last result no longer holds if we drop the assumption that STS is step-finite. Consider, for example, $S T S=\left(\left\{s_{0}, s_{1}, \ldots\right\},\{a, b\}, \rightarrow, s_{0}\right)$, where:

$$
\rightarrow=\left\{\left(s_{i}, a^{j}, s_{i}\right) \mid i \geq 0 \wedge j \leq i\right\} \cup\left\{\left(s_{i}, b+a^{j}, s_{i+1}\right) \mid i \geq 0 \wedge j \leq i\right\} .
$$

Then $S T S^{m r e v}$ is solvable by the PT-net in Figure $3(b)$, but $S T S^{\text {srev }}$ is not solvable by any PT-net $N=\left(P,\{a, b, \bar{a}, \bar{b}\}, F, M_{0}\right)$. Indeed, if $N$ existed, then it would have distinct reachable markings $M_{0}, M_{1}, \ldots$ such that, for all $i \geq 0$ :

(i) $M_{i} \stackrel{b}{\longrightarrow} M_{i+1}$, (ii) $M_{i} \stackrel{a^{i}}{\longrightarrow} N M_{i}, \quad$ (iii) $M_{i} \stackrel{\bar{a}}{\longrightarrow} M_{N} M_{i}$ (for $i>0$ ), but we would not have (iv) $M_{i} \stackrel{(a \bar{a})}{\longrightarrow}_{N} M_{i}$.

We now observe that $(i)$ means that $M_{0} \leq M_{1} \leq \ldots$ Hence, $(i v)$ together with the finiteness of $P$, implies that there is $p \in P$ such that $F(p, a)+F(p, \bar{a})>$ $M_{0}(p)=M_{1}(p)=\ldots$ But (iii) implies $F(p, \bar{a}) \leq M_{0}(p)=M_{1}(p)=\ldots$, and from (ii) we obtain $F(p, a)=0$, getting $F(p, a)+F(p, \bar{a}) \leq M_{0}(p)$, which contradicts our first inequation.

Corollary 2. Let STS be a well-formed set transition system. If STS ${ }^{m r e v}$ is solvable, then $S T S^{\text {rev }}$ is also solvable.

Proof. As a set transition system, STS is step-finite and $S T S^{\text {rev }}=S T S^{\text {srev }}$. Hence the result follows from Proposition 7.

\section{$5 \quad$ Reversibility and plain solvability}

The feasibility of reversing steps in WFST-systems can in some cases be replaced by checking the solvability of the original transition system, and the solvability of its pure reversed version(s). The latter are formalised in the following way.

Let $S T S=\left(S, T, \rightarrow, s_{0}\right)$ be a WFST-system and $r \in S$.

Then we define the step transition system $\overline{S T S}_{r}=\left(\operatorname{pred}(r), \bar{T}, \rightarrow_{r}, r\right)$, where:

$$
\rightarrow_{r}=\left\{\left(s^{\prime}, \bar{\alpha}, s\right) \mid s^{\prime} \in \operatorname{pred}(r) \wedge s \stackrel{\alpha}{\longrightarrow} s^{\prime}\right\} .
$$

It is easy to check that $\overline{S T S}_{r}$ is also well-formed. Moreover, since $S T S$ satisfies $R E A, s_{0} \in \operatorname{pred}(r)$ and it is reachable in $\overline{S T S}_{r}$ from every state of the latter.

Theorem 1. Let $R$ be a home cover of a WFST-system STS. Then STS ${ }^{m r e v}$ is solvable iff STS is solvable and $\overline{S T S}_{r}$ (for all $r \in R$ ) are simultaneously solvable. 
Proof. Note that $S=\bigcup_{r \in R} S_{r}$, as $R$ is a home cover. In the proof below, we will use the following notation, where $r \in R$ :

$$
\begin{array}{lll}
S T S & =\left(S, T, \rightarrow, s_{0}\right) & S T S^{r e v}=\left(S, T \cup \bar{T}, \rightarrow_{r e v}, s_{0}\right) \\
S T S^{\text {mrev }}=\left(S, T \cup \bar{T}, \rightarrow_{\text {mrev }}, s_{0}\right) & \overline{S T S}_{r}=\left(S_{r}, \bar{T}, \rightarrow_{r}, r\right) .
\end{array}
$$

$(\Longrightarrow)$ Suppose that $N=\left(P, T, F, M_{0}\right)$ is such that $S T S^{\text {mrev }} \simeq_{\psi} C R G_{N}$.

To show that $S T S$ is solvable, let $N^{\prime}=\left.N\right|_{T}$. Then $S T S \simeq_{\psi} C R G_{N^{\prime}}$. Indeed, we first note that $\psi\left(s_{0}\right)=M_{0}$. Suppose now that $s \in S$ and $\psi(s) \in$ reach $_{N^{\prime}}$. Let us see that the execution of transitions is preserved in both directions by $\psi$ :

i) $s \stackrel{\alpha}{\longrightarrow} s^{\prime}$. Then, by Proposition $2(2)$, we have $s \stackrel{\alpha}{\longrightarrow}$ mrev $s^{\prime}$. Hence, by $S T S^{\text {mrev }} \simeq_{\psi} C R G_{N}$, we have $\psi(s) \stackrel{\alpha}{\longrightarrow}{ }_{N} \psi\left(s^{\prime}\right)$. Moreover, the enabling and firing of steps over $T$ are exactly the same in $N$ and $N^{\prime}$. Hence $\psi(s) \stackrel{\alpha}{\longrightarrow} N^{\prime} \psi\left(s^{\prime}\right)$.

ii) $\psi(s) \stackrel{\alpha}{\longrightarrow} N^{\prime} M$. Then, as the enabling and firing of steps over $T$ are exactly the same in $N$ and $N^{\prime}, \psi(s) \stackrel{\alpha}{\longrightarrow}{ }_{N} M$. Hence, by $S T S^{m r e v} \simeq_{\psi} C R G_{N}$, $M \in \psi(S)$ and $s \stackrel{\alpha}{\longrightarrow}$ mrev $\psi^{-1}(M)$. Thus, by Proposition $2(2), s \stackrel{\alpha}{\longrightarrow} \psi^{-1}(M)$.

To show that the $\overline{S T S}_{r}$ 's are simultaneously solvable, let us take $N_{r}$ as the net $\left.N\right|_{\bar{T}}$ with the initial marking set to $\psi(r)$, for every $r \in R$. Then $\overline{S T S}_{r} \simeq_{\psi}$ $C R G_{N_{r}}$. Indeed, we first note that the initial states of $\overline{S T S}$ r and $C R G_{N_{r}}$ are related by $\psi$. Suppose now that $s$ is a state in $\overline{S T S}_{r}$ such that $\psi(s) \in$ reach $_{N_{r}}$. Again we have:

i) $s \stackrel{\bar{\alpha}}{\longrightarrow} s^{\prime}$. Then $s \stackrel{\bar{\alpha}}{\longrightarrow}$ rev $s^{\prime}$ and so, by Proposition $2(3)$, we have $s \stackrel{\bar{\alpha}}{\longrightarrow}$ mrev $s^{\prime}$. Hence we have, by $S T S^{m r e v} \simeq_{\psi} C R G_{N}, \psi(s) \stackrel{\bar{\alpha}}{\longrightarrow}{ }_{N} \psi\left(s^{\prime}\right)$. Moreover, the enabling and firing of steps over $\bar{T}$ are exactly the same in $N$ and $N_{r}$. Hence $\psi(s) \stackrel{\bar{\alpha}}{\longrightarrow} N_{r} \psi\left(s^{\prime}\right)$.

ii) $\psi(s) \stackrel{\bar{\alpha}}{\longrightarrow} N^{\prime} M$. Then, as the enabling and firing of steps over $\bar{T}$ are exactly the same in $N$ and $N_{r}$, we have $\psi(s) \stackrel{\bar{\alpha}}{\longrightarrow}{ }_{N} M$. Hence, by $S T S^{\text {mrev }} \simeq_{\psi} C R G_{N}$, we have $M \in \psi(S)$ and $s \stackrel{\bar{\alpha}}{\longrightarrow} S_{S S^{m r e v}} \psi^{-1}(M)$. Thus, by Proposition $2(3), s \stackrel{\bar{\alpha}}{\longrightarrow} r$ $\psi^{-1}(M)$.

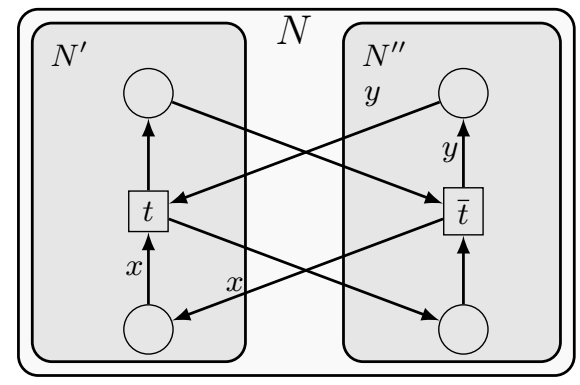

Fig. 4. An illustration of the proof of Theorem 1.

$(\Longleftarrow)$ Since $S T S$ is solvable, there is a PT-net $N^{\prime}=\left(P^{\prime}, T, F^{\prime}, M_{0}^{\prime}\right)$ such that $S T S \simeq_{\psi^{\prime}} C R G_{N^{\prime}}$. And, since the $\overline{S T S}_{r}$ 's are simultaneously solvable, there are PT-nets $N_{r}=\left(P^{\prime \prime}, \bar{T}, F^{\prime \prime}, M_{r}\right)$ (for all $r \in R$ ) and $\psi^{\prime \prime}: S \rightarrow \bigcup_{r \in R}$ reach $_{N_{r}}$ 
such that $\overline{S T S}_{r} \simeq_{\psi^{\prime \prime}} C R G_{N_{r}}$, for every $r \in R$. Note that $\psi^{\prime}\left(s_{0}\right)=M_{0}^{\prime}$ and $\psi^{\prime \prime}(r)=M_{r}$, for every $r \in R$. Clearly, we may assume that $P^{\prime} \cap P^{\prime \prime}=\varnothing$.

Let $N=\left(P^{\prime} \cup P^{\prime \prime}, T \cup \bar{T}, F, M_{0}\right)$ the PT-net, where $M_{0}=M_{0}^{\prime}+\psi^{\prime \prime}\left(s_{0}\right)=$ $\psi^{\prime}\left(s_{0}\right)+\psi^{\prime \prime}\left(s_{0}\right)$. Now taking $N^{\prime \prime}=\left(P^{\prime \prime}, \bar{T}, F^{\prime \prime}, \varnothing\right)$, for every $t \in T$ we have:

$$
\begin{array}{ll}
\operatorname{pre}_{N}(t)=\operatorname{pre}_{N^{\prime}}(t)+\text { post }_{N^{\prime \prime}}(\bar{t}) & \text { post }_{N}(t)=\text { post }_{N^{\prime}}(t)+\text { pre }_{N^{\prime \prime}}(\bar{t}) \\
\operatorname{pre}_{N}(\bar{t})=\operatorname{pre}_{N^{\prime \prime}}(\bar{t})+\text { post }_{N^{\prime}}(t) & \text { post }_{N}(\bar{t})=\text { post }_{N^{\prime \prime}}(\bar{t})+\text { pre }_{N^{\prime}}(t) .
\end{array}
$$

Note that $N$ is a PT-net with strict reverses (see Figure 4). Moreover, for all $t \in T$ and $r \in R$ :

$$
\operatorname{pre}_{N^{\prime \prime}}(\bar{t})=\operatorname{pre}_{N_{r}}(\bar{t}) \quad \operatorname{post}_{N^{\prime \prime}}(\bar{t})=\operatorname{post}_{N_{r}}(\bar{t}) .
$$

Let $\psi$ be a mapping with the domain $S$ which, for every $s \in S$, returns $\psi^{\prime}(s)+\psi^{\prime \prime}(s)$. Note that $\psi$ is well-defined since $R$ is a home cover of STS, and that $\psi\left(s_{0}\right)=M_{0}$.

We first show that $S T S^{r e v} \simeq_{\psi} S T S^{\prime}$, where $S T S^{\prime}$ is just $C R G_{N}$ but removing from it all the arcs labelled by the mixed steps (i.e., steps of the form $\alpha+\bar{\beta}$, for $\alpha, \beta \neq \varnothing$ ) deleted. (Note that this does not produce unreachable states since $C R G_{N}$ satisfies $S E Q$.) Indeed, we first note that the initial states of $S T S^{r e v}$ and $S T S^{\prime}$ are related by $\psi$. Suppose now that $s \in S$ and $\psi(s) \in$ reach $_{N}$. Once again we see that the execution of transitions is preserved in both directions by $\psi$ :

i) $s \stackrel{\alpha}{\longrightarrow}$ rev $s^{\prime}$. Then, by $S T S \simeq_{\psi^{\prime}} C R G_{N^{\prime}}$, we have $\psi^{\prime}(s) \stackrel{\alpha}{\longrightarrow} N_{N^{\prime}} \psi^{\prime}\left(s^{\prime}\right)$. Moreover, $s^{\prime} \stackrel{\bar{\alpha}}{\longrightarrow}$ rev $s$. Hence, since $R$ is a home cover, there is $r \in R$ such that $s^{\prime} \stackrel{\bar{\alpha}}{\longrightarrow}_{r} s$. Thus, by $\overline{S T S}_{r} \simeq_{\psi^{\prime \prime}} C R G_{N_{r}}$, we have $\psi^{\prime \prime}\left(s^{\prime}\right) \stackrel{\bar{\alpha}}{\longrightarrow}_{N_{r}} \psi^{\prime \prime}(s)$, and so $\psi^{\prime \prime}(s) \geq$ post $_{N_{r}}(\bar{\alpha})$. Hence, by (2) and (3), we have:

$$
\psi(s)=\psi^{\prime}(s)+\psi^{\prime \prime}(s) \geq \operatorname{pre}_{N^{\prime}}(\alpha)+\text { post }_{N_{r}}(\bar{\alpha})=\operatorname{pre}_{N}(\alpha) .
$$

As a result, $\psi(s) \stackrel{\alpha}{\longrightarrow} \psi(s)+$ eff $_{N}(\alpha)$. Moreover, by (2) and (3), we have:

$$
\begin{aligned}
\psi(s)+ & \operatorname{eff}_{N}(\alpha) \\
& =\psi^{\prime}(s)+\psi^{\prime \prime}(s)+\operatorname{post}_{N}(\alpha)-\operatorname{pre}_{N}(\alpha) \\
& =\psi^{\prime}(s)+\psi^{\prime \prime}(s)+\left(\operatorname{post}_{N^{\prime}}(\alpha)+\operatorname{pre}_{N^{\prime \prime}}(\bar{\alpha})\right)-\left(\operatorname{pre}_{N^{\prime}}(\alpha)+\operatorname{post}_{N^{\prime \prime}}(\bar{\alpha})\right) \\
& =\left(\psi^{\prime}(s)+\operatorname{post}_{N^{\prime}}(\alpha)-\operatorname{pre}_{N^{\prime}}(\alpha)\right)+\left(\psi^{\prime \prime}(s)-\operatorname{post}_{N^{\prime \prime}}(\bar{\alpha})+\operatorname{pre}_{N^{\prime \prime}}(\bar{\alpha})\right) \\
& =\psi^{\prime}\left(s^{\prime}\right)+\psi^{\prime \prime}\left(s^{\prime}\right) .
\end{aligned}
$$

Hence $\psi(s) \stackrel{\alpha}{\longrightarrow} \psi\left(s^{\prime}\right)$.

ii) $s \stackrel{\bar{\alpha}}{\longrightarrow}$ rev $s^{\prime}$. Then $s^{\prime} \stackrel{\alpha}{\longrightarrow}_{\text {rev }} s$ and so, by Case $1, \psi\left(s^{\prime}\right) \stackrel{\alpha}{\longrightarrow}_{N} \psi(s)$. Hence, since $N$ is PT-net with strict reverses, $\psi(s) \stackrel{\bar{\alpha}}{\longrightarrow}{ }_{N} \psi\left(s^{\prime}\right)$.

iii) $\psi(s) \stackrel{\alpha}{\longrightarrow} N$. Then

$$
\begin{gathered}
\psi^{\prime}(s)+\psi^{\prime \prime}(s)=\psi(s) \geq \operatorname{pre}_{N}(\alpha)=\operatorname{pre}_{N^{\prime}}(\alpha)+\operatorname{post}_{N^{\prime \prime}}(\bar{\alpha}) . \\
M=\psi^{\prime}(s)+\psi^{\prime \prime}(s)+\operatorname{post}_{N^{\prime}}(\alpha)+\operatorname{pre}_{N^{\prime \prime}}(\bar{\alpha})-\left(\operatorname{pre}_{N^{\prime}}(\alpha)+\operatorname{post}_{N^{\prime \prime}}(\bar{\alpha})\right) .
\end{gathered}
$$


Hence, since $P^{\prime} \cap P^{\prime \prime}=\varnothing, \psi^{\prime}(s) \geq \operatorname{pre}_{N^{\prime}}(\alpha)$ and $\psi^{\prime \prime}(s) \geq$ post $_{N^{\prime \prime}}(\bar{\alpha})$. Moreover, we have:

$$
\begin{aligned}
& M \cap P^{\prime}=\psi^{\prime}(s)+\operatorname{post}_{N^{\prime}}(\alpha)-\text { pre }_{N^{\prime}}(\alpha) \\
& M \cap P^{\prime \prime}=\psi^{\prime \prime}(s)+\operatorname{pre}_{N^{\prime \prime}}(\bar{\alpha})-\text { post }_{N^{\prime \prime}}(\bar{\alpha}) .
\end{aligned}
$$

Thus $\psi^{\prime}(s) \stackrel{\alpha}{\longrightarrow} N^{\prime} M \cap P^{\prime}$. Hence, by $S T S \simeq_{\psi^{\prime}} C R G_{N^{\prime}}$, we obtain $M \cap P^{\prime} \in \psi^{\prime}(S)$ and $s \stackrel{\alpha}{\longrightarrow}$ rev $s^{\prime}$, where $\psi^{\prime}\left(s^{\prime}\right)=M \cap P^{\prime}$. We need to show that $\psi(s)=M$, and this would follow from $\psi^{\prime \prime}\left(s^{\prime}\right)=M \cap P^{\prime \prime}$. Indeed, we have $s^{\prime} \stackrel{\bar{\alpha}}{\longrightarrow}$ rev $s$, and so there is $r \in R$ such that $s^{\prime} \in S_{r}$. Now, by $\overline{S T S}_{r} \simeq_{\psi^{\prime \prime}} C R G_{N_{r}}, \psi^{\prime \prime}\left(s^{\prime}\right) \stackrel{\bar{\alpha}}{\longrightarrow} N_{r} \psi^{\prime \prime}(s)$. But this means that $\psi^{\prime \prime}(s)=\psi^{\prime \prime}\left(s^{\prime}\right)+$ post $_{N^{\prime \prime}}(\bar{\alpha})-$ pre $_{N^{\prime \prime}}(\bar{\alpha})$. Thus

$$
\psi^{\prime \prime}\left(s^{\prime}\right)=\psi^{\prime \prime}(s)-\text { post }_{N^{\prime \prime}}(\bar{\alpha})+\text { pre }_{N^{\prime \prime}}(\bar{\alpha})=M \cap P^{\prime \prime} .
$$

iv) $\psi(s) \stackrel{\bar{\alpha}}{\longrightarrow} N$. Then we have:

$$
\begin{gathered}
\psi^{\prime}(s)+\psi^{\prime \prime}(s)=\psi(s) \geq \operatorname{pre}_{N}(\bar{\alpha})=\operatorname{pre}_{N^{\prime \prime}}(\bar{\alpha})+\operatorname{post}_{N^{\prime}}(\alpha) \\
M=\psi^{\prime}(s)+\psi^{\prime \prime}(s)+\text { post }_{N^{\prime \prime}}(\bar{\alpha})+\operatorname{pre}_{N^{\prime}}(\alpha)-\left(\operatorname{pre}_{N^{\prime \prime}}(\bar{\alpha})+\operatorname{post}_{N^{\prime}}(\alpha)\right) .
\end{gathered}
$$

Hence, since $P^{\prime} \cap P^{\prime \prime}=\varnothing, \psi^{\prime}(s) \geq$ post $_{N^{\prime}}(\alpha)$ and $\psi^{\prime \prime}(s) \geq$ pre $_{N^{\prime \prime}}(\bar{\alpha})$. Moreover, we have:

$$
\begin{aligned}
& M \cap P^{\prime}=\psi^{\prime}(s)+\operatorname{pre}_{N^{\prime}}(\alpha)-\text { post }_{N^{\prime}}(\alpha) \\
& M \cap P^{\prime \prime}=\psi^{\prime \prime}(s)+\text { post }_{N^{\prime \prime}}(\bar{\alpha})-\operatorname{pre}_{N^{\prime \prime}}(\bar{\alpha}) .
\end{aligned}
$$

Thus $\psi^{\prime \prime}(s) \stackrel{\bar{\alpha}}{\longrightarrow} N^{\prime \prime} M \cap P^{\prime \prime}$. Hence, since $R$ is a home cover, there is $r \in R$ such that $s \in S_{r}$. Thus, by $\overline{S T S}_{r} \simeq_{\psi^{\prime \prime}} C R G_{N_{r}}, M \cap P^{\prime \prime} \in \psi^{\prime \prime}(S)$ and $s \stackrel{\bar{\alpha}}{\longrightarrow}$ rev $s^{\prime}$, where $\psi^{\prime \prime}\left(s^{\prime}\right)=M \cap P^{\prime \prime}$. We need to show that $\psi(s)=M$, and this would follow from $\psi^{\prime}\left(s^{\prime}\right)=M \cap P^{\prime}$. Indeed, we have $s^{\prime} \stackrel{\bar{\alpha}}{\longrightarrow}$ rev $s$. Hence, by $S T S \simeq_{\psi^{\prime}} C R G_{N^{\prime}}$, we obtain $\psi^{\prime}\left(s^{\prime}\right) \stackrel{\bar{\alpha}}{\longrightarrow} N^{\prime} \psi^{\prime}(s)$. But this means that

$$
\psi^{\prime}(s)=\psi^{\prime}\left(s^{\prime}\right)+\text { post }_{N^{\prime}}(\alpha)-\operatorname{pre}_{N^{\prime}}(\alpha),
$$

and so we obtain: $\psi^{\prime}\left(s^{\prime}\right)=\psi^{\prime}(s)-$ post $_{N^{\prime}}(\alpha)+\operatorname{pre}_{N^{\prime}}(\alpha)=M \cap P^{\prime}$.

Now in order to conclude $S T S^{m r e v} \simeq_{\psi} C R G_{N}$ we only need to consider the case of mixed transitions:

i) $s \stackrel{\alpha+\beta}{\longrightarrow}$ rev and $s \oplus \alpha \stackrel{\bar{\alpha}+\beta}{\longrightarrow}$ mrev $s \oplus \beta$. Then $s \stackrel{\alpha}{\longrightarrow}_{r e v} s \oplus \alpha$ and $s \stackrel{\beta}{\longrightarrow}$ rev $s \oplus \beta$. Thus, by $S T S^{r e v} \simeq_{\psi} S T S^{\prime}$,

$$
\psi(s) \stackrel{\alpha+\beta}{\longrightarrow} N \quad \psi(s) \stackrel{\alpha}{\longrightarrow} \text { rev } \psi(s \oplus \alpha) \quad \psi(s) \stackrel{\beta}{\longrightarrow}_{r e v} \psi(s \oplus \beta) .
$$

Hence, we have $\psi(s) \geq \operatorname{pre}_{N}(\alpha+\beta)=\operatorname{pre}_{N}(\alpha)+\operatorname{pre}_{N}(\beta)$. Thus

$$
\begin{aligned}
& \psi(s) \stackrel{\alpha}{\longrightarrow}{ }_{N} \psi(s)+\text { eff }_{N}(\alpha)=\psi(s)+\operatorname{post}_{N}(\alpha)-\operatorname{pre}_{N}(\alpha) \\
& =\psi(s)+\operatorname{pre}_{N}(\bar{\alpha})-\operatorname{pre}_{N}(\alpha) \geq \operatorname{pre}_{N}(\bar{\alpha}+\beta) .
\end{aligned}
$$

Moreover, by $F D, \psi(s \oplus \alpha)=\psi(s)+\operatorname{eff}_{N}(\alpha) \geq \operatorname{pre}_{N}(\bar{\alpha}+\beta)$. And, finally, $\psi(s)+$ eff $_{N}(\alpha)+$ eff ${ }_{N}(\bar{\alpha}+\beta)=\psi(s)+$ eff $_{N}(\beta)$. 
ii) $\psi(s) \stackrel{\bar{\alpha}+\beta}{\longrightarrow} N M$. Then we have $\psi(s) \stackrel{\bar{\alpha}}{\longrightarrow}{ }_{N} \psi(s) \oplus \bar{\alpha}$ and, using that $\operatorname{post}(\bar{\alpha})=\operatorname{pre}_{N}(\alpha), \psi(s) \oplus \bar{\alpha} \stackrel{\alpha+\beta}{\longrightarrow}$. Thus, from $S T S^{\text {rev }} \simeq_{\psi} S T S^{\prime}$ it follows that $\psi^{-1}(\psi(s) \oplus \bar{\alpha}) \stackrel{\bar{\alpha}+\beta}{\longrightarrow}$ rev . Hence

$$
\psi^{-1}(\psi(s) \oplus \bar{\alpha}) \oplus \alpha \stackrel{\bar{\alpha}+\beta}{\longrightarrow}_{\text {mrev }} \psi^{-1}(\psi(s) \oplus \bar{\alpha}) \oplus \beta .
$$

All we need to show now is that:

$$
\begin{aligned}
\psi^{-1}(\psi(s) \oplus \bar{\alpha}) \oplus \alpha & =s \\
\left.\psi\left(\psi^{-1}(\psi(s) \oplus \bar{\alpha}) \oplus \beta\right)\right) & =\psi(s) \oplus(\bar{\alpha}+\beta),
\end{aligned}
$$

which clearly is the case.

Corollary 3. Let $r$ be a home state of a WFST-system STS. Then STS ${ }^{m r e v}$ is solvable iff STS and $\overline{S T S}_{r}$ are solvable.

The above corollary and the proof of the last theorem provide a method for constructing a PT-net implementing mixed step reversibility provided that one can synthesise PT-nets for two step transition systems using, e.g., theory of regions $[1,10]$.

We have obtained a method for checking the feasibility of mixed reversability. This is indeed useful, in view of Proposition 6. Moreover, for set transition systems the result extends to direct reversibility.

Theorem 2. Let $r$ be a home state of a well-formed set transition system STS. Then STS ${ }^{\text {rev }}$ is solvable iff STS and $\overline{S T S}_{r}$ are solvable.

Proof. $(\Longrightarrow)$ Let $S T S^{r e v} \simeq_{\psi} C R G_{N}$ and $T$ be the set of transitions of $N$. Then $S T S \simeq_{\psi} C R G_{\left.N\right|_{T}}$ and $\overline{S T S}_{r} \simeq_{\psi} C R G_{N^{\prime}}$, where $N^{\prime}$ is $\left.N\right|_{\bar{T}}$ with the initial marking set to $\psi(r)$.

$(\Longleftarrow)$ Follows from Theorem 1 and Corollary 2 .

\section{From sequential reversibility to step reversibility}

Checking the feasibility of step reversibility and then constructing a suitable PT-net can be difficult. Our next result shows that in certain cases one can carry out this task more easily, if we are given a net that simultaneously solves the original transition system, overapproximates its reversed version that contains only spikes, and underapproximates its mixed reversed version.

Theorem 3. Let $N=\left(P, T \cup \bar{T}, F, M_{0}\right)$ be a PT-net, and $S T S=\left(S, T, \rightarrow, s_{0}\right)$ be a WFST-system such that:

$$
\begin{aligned}
\left(S T S^{\text {spike }}\right)^{\text {rev }} & \triangleleft C R G_{N} \triangleleft S T S^{\text {mrev }} \\
S T S & \simeq C R G_{N_{T}} .
\end{aligned}
$$

Then STS ${ }^{\text {mrev }}$ is solvable. Moreover, if STS is a set transition system, then STS $^{\text {rev }}$ is solvable. 
Proof. The states as well as the initial states of $\left(S T S^{\text {spike }}\right)^{\text {rev }}, S T S^{\text {mrev }}$, and $S T S$ are all the same; moreover, $\left(\left.\left(S T S^{\text {spike }}\right)^{r e v}\right|_{T}\right)^{\text {seq }}=\left(\left.S T S^{\text {mrev }}\right|_{T}\right)^{\text {seq }}=S T S^{\text {seq }}$. Similarly, the initial states of $C R G_{N}$ and $C R G_{\left.N\right|_{T}}$ are the same and we have $\left.\left(C R G_{N}\right)\right|_{T}=C R G_{\left.N\right|_{T}}$.

Moreover, all transition systems in (4) and (5) satisfy FD $\& R E A \& S E Q$, and there is a bijection $\psi$ such that:

$$
\left(S T S^{\text {spike }}\right)^{r e v} \triangleleft_{\psi} C R G_{N} \triangleleft_{\psi^{-1}} S T S^{\text {mrev }} \text { and } S T S \simeq_{\psi} C R G_{\left.N\right|_{T}} .
$$

By (4) and $S E Q$ of step transition systems and reachability graphs and the fact that we may assume that each $t \in T$ appears in the labels of the arcs of $S T S$, we have for any $t \in T$ :

$$
\operatorname{reach}_{N}=\operatorname{reach}_{\left.N\right|_{T}} \text { and eff }{ }_{N}(t)=-e f f_{N}(\bar{t}) .
$$

We first show that it can be assumed that, for all $t \in T$ :

$$
\operatorname{pre}_{N}(\bar{t}) \geq \operatorname{post}_{N}(t) \text { and } \text { post }_{N}(\bar{t}) \geq \operatorname{pre}_{N}(t) \text {. }
$$

Indeed, suppose that $F(p, \bar{t})<F(t, p)$. We then modify $F$ to become $F^{\prime}$ which is the same as $F$ except that $F^{\prime}(p, \bar{t})=F(t, p)$ and $F^{\prime}(\bar{t}, p)=F(p, t)$. Let $N^{\prime}$ be the resulting PT-net. Clearly, eff ${ }_{N}(x)=$ eff $_{N^{\prime}}(x)$, for every $x \in T \cup \bar{T}$.

After this modification - which does not affect transitions in $T-(5)$ is still satisfied after taking $N^{\prime}$ to play the role of $N$. However, the satisfaction of (4) is not so immediate. But the modification can only restrict the enabling of steps, and the enabling of transitions other than $\bar{t}$ is unchanged. Thus

$$
C R G_{N^{\prime}} \triangleleft C R G_{N} \triangleleft S T S^{m r e v} .
$$

Hence, if (4) does not hold with $N^{\prime}$ playing the role of $N$, then there is $M \in$ reach $_{N^{\prime}} \subseteq$ reach $_{N}$ and $k \geq 1$ such that:

$$
M \stackrel{\bar{t}^{k}}{\longrightarrow}{ }_{C R G_{N}} M^{\prime} \text { and } \neg M \stackrel{\bar{t}^{k}}{\longrightarrow}{ }_{C R G_{N^{\prime}}} .
$$

By (4) and the first part of (9), we have:

$$
M^{\prime} \stackrel{t^{k}}{\longrightarrow}{C R G_{N}} M \text { and so } M(p) \geq F\left(t^{k}, p\right) .
$$

By construction, the only reason for the second part of (9) to hold is that $F^{\prime}\left(p, \bar{t}^{k}\right)>M(p)$. Thus, by $F^{\prime}\left(p, \bar{t}^{k}\right)=F\left(t^{k}, p\right)$, we obtain $F\left(t^{k}, p\right)>M(p)$, yielding a contradiction with (10).

We can apply the above modification as many times as needed, finally concluding that (8) can be assumed to hold for $N$ as any modification does not invalidate the conditions captured by (8) that were got by the previous modifications.

We next show that $S T S^{m r e v}$ is solvable, after constructing a PT-net $\widetilde{N}=$ $\left(\widetilde{P}, T \cup \bar{T}, \widetilde{F}, \widetilde{M}_{0}\right)$, in the following way: 


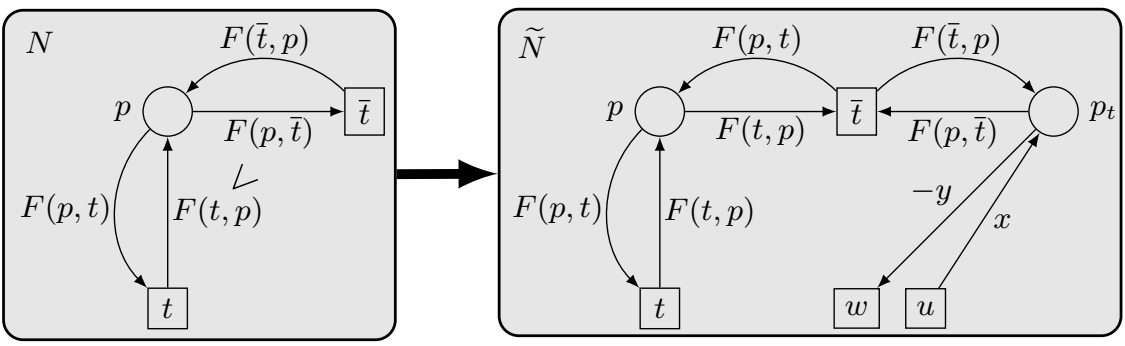

Fig. 5. Constructing place $p_{t}$ in the proof of Theorem 3, where $x=$ eff $_{N}(u)(p)>0$ and $y=\operatorname{eff}_{N}(w)(p) \leq 0$. Note that $u, w \in T \cup \bar{T} \backslash\{\bar{t}\}$.

- $\widetilde{P}=\bigcup_{p \in P} P_{p}$, where, for every $p \in P{ }^{8}$

$$
P_{p}=\{p\} \cup\left\{p_{t} \mid t \in T \wedge F(p, \bar{t})>F(t, p)\right\} \text { and } \widetilde{M}_{0}\left(P_{p}\right)=\left\{M_{0}(p)\right\} .
$$

- The connections in $\tilde{N}$ are set as follows, where $p \in P$ and $u \in T \cup \bar{T} \backslash\{\bar{t}\}$ :

- $\widetilde{F}(p, \bar{t})=F(t, p)$ and $\widetilde{F}(\bar{t}, p)=F(p, t)$.

- $\widetilde{F}\left(p_{t}, \bar{t}\right)=F(p, \bar{t})$ and $\widetilde{F}\left(\bar{t}, p_{t}\right)=F(\bar{t}, p)$.

- eff $_{N}(u)(p)>0$ implies $\widetilde{F}\left(p_{t}, u\right)=0$ and $\widetilde{F}\left(u, p_{t}\right)=$ eff $_{N}(u)(p)$.

- eff ${ }_{N}(u)(p) \leq 0$ implies $\widetilde{F}\left(u, p_{t}\right)=0$ and $\widetilde{F}\left(p_{t}, u\right)=-e f f_{N}(u)(p)$.

- $\widetilde{F}$ on $(P \times T) \cup(T \times P)$ is as $F$ unless it has been set explicitly above.

In what follows, for every marking $M$ of $N$, we use $\phi(M)$ to denote the marking of $\widetilde{N}$ such that $\phi(M)\left(P_{p}\right)=\{M(p)\}$, for every $p \in P$. Hence $\phi\left(M_{0}\right)=\widetilde{M}_{0}$.

We now present a number of straightforward properties of $\widetilde{N}$. We first observe that, by (8), for all $t \in T, u \in T \cup \bar{T}$, and $p \in P$,

$$
\begin{aligned}
\operatorname{pre}_{\widetilde{N}}(\bar{t}) & \geq \operatorname{post}_{\widetilde{N}}(t) & \text { eff }_{\widetilde{N}}(t) & =- \text { eff }_{\widetilde{N}}(\bar{t}) \\
\operatorname{post}_{\widetilde{N}}(\bar{t}) \geq \operatorname{pre}_{\widetilde{N}}(t) & \text { eff } \widetilde{N} & (u)\left(P_{p}\right) & =\left\{\text { eff }_{N}(u)(p)\right\} .
\end{aligned}
$$

Therefore, for every marking $M$ of $N$ and every $\kappa \in \operatorname{mult}(T \cup \bar{T})$ such that $M+e f f_{N}(\kappa) \geq \varnothing$,

$$
\phi(M)+e f f_{\widetilde{N}}(\kappa)=\phi\left(M+e f f_{N}(\kappa)\right) .
$$

The construction does not affect the enabling of steps involving just one transition as well as steps $\alpha$ over $T$ since $p_{t} \in P_{p}$ cannot disable $\alpha$ if it is not also disabled by $p$. Hence, for all markings $M$ of $N, u \in T \cup \bar{T}, k \geq 1$, and $\alpha \in \operatorname{mult}(T)$,

$$
M\left[u^{k}\right\rangle_{N} \Longleftrightarrow \phi(M)\left[u^{k}\right\rangle_{\widetilde{N}} \text { and } M[\alpha\rangle_{N} \Longleftrightarrow \phi(M)[\alpha\rangle_{\widetilde{N}} .
$$

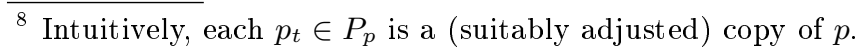


Hence, by $(6,12,13)$ and $\widetilde{M}_{0}=\phi\left(M_{0}\right)$,

$\left(S T S^{\text {spike }}\right)^{\text {rev }} \triangleleft_{\phi \circ \psi} C R G_{\widetilde{N}}$ and $S T S \simeq_{\phi \circ \psi} C R G_{\left.\widetilde{N}\right|_{T}} \simeq_{\phi^{-1}} C R G_{N_{\left.\right|_{T}}}$.

We then show that, for every marking $M$ of $\widetilde{N}$ and all $\alpha, \beta \in \operatorname{mult}(T)$ :

(A) $\phi(M) \stackrel{\bar{\alpha}+\beta}{\longrightarrow} \widetilde{N}_{\text {implies }} \phi(M)-$ eff $_{\widetilde{N}}(\alpha) \stackrel{\alpha+\beta}{\longrightarrow} \widetilde{N} \phi(M)+$ eff $_{\widetilde{N}}(\beta)$. Indeed, we first observe that $\phi(M)-$ eff $_{\widetilde{N}}(\alpha) \in \operatorname{reach}_{\widetilde{N}}$. We then observe that, by $\phi(M) \geq \operatorname{pre}_{\widetilde{N}}(\bar{\alpha}+\beta)$, we have:

$$
\begin{aligned}
\phi(M)-\operatorname{eff}_{\widetilde{N}}(\alpha) & \geq \quad \operatorname{pre}_{\widetilde{N}}(\bar{\alpha}+\beta)-\operatorname{eff}_{\widetilde{N}}(\alpha) \\
& =\quad \operatorname{pre}_{\widetilde{N}}(\bar{\alpha})+\operatorname{pre}_{\widetilde{N}}(\beta)-\operatorname{post}_{\widetilde{N}}(\alpha)+\operatorname{pre}_{\widetilde{N}}(\alpha) \\
& \geq \text { by (11) } \operatorname{pre}_{\widetilde{N}}(\alpha+\beta) .
\end{aligned}
$$

Hence $\alpha+\beta$ is enabled at $\phi(M)-\operatorname{eff}_{\widetilde{N}}(\alpha)$, and $(A)$ holds as we have:

$$
\phi(M)-\operatorname{eff}_{\widetilde{N}}(\alpha)+\operatorname{eff}_{\widetilde{N}}(\alpha+\beta)=\phi(M)+\operatorname{eff}_{\widetilde{N}}(\beta) .
$$

(B) $\phi(M) \stackrel{\alpha+\beta}{\longrightarrow} \widetilde{N}$ implies $\phi(M)+\operatorname{eff}_{\widetilde{N}}(\alpha) \stackrel{\bar{\alpha}+\beta}{\longrightarrow} \widetilde{N} \phi(M)+$ eff $_{\widetilde{N}}(\beta)$. Indeed, by $S E Q$ of reachability graphs, $\phi(M) \stackrel{\alpha}{\longrightarrow} \tilde{N} \phi(M)+e f \widetilde{N}_{\widetilde{N}}(\alpha)=M^{\prime}$. Suppose that $M^{\prime} \stackrel{\bar{\alpha}+\beta}{\longrightarrow} \widetilde{N}$ does not hold. Then there is $q \in \widetilde{P}$ such that

$$
\widetilde{F}(q, \bar{\alpha}+\beta)>M^{\prime}(q) .
$$

Moreover, $\phi(M)(q) \geq \widetilde{F}(q, \alpha+\beta)$ and $M^{\prime}(q)=\phi(M)(q)-\widetilde{F}(q, \alpha)+\widetilde{F}(\alpha, q)$. Hence:

$$
\begin{aligned}
\widetilde{F}(q, \bar{\alpha}+\beta) & >\phi(M)(q)-\widetilde{F}(q, \alpha)+\widetilde{F}(\alpha, q) \\
& \geq \widetilde{F}(q, \alpha+\beta)-\widetilde{F}(q, \alpha)+\widetilde{F}(\alpha, q),
\end{aligned}
$$

and so, by erasing $\widetilde{F}(q, \beta)$ from both sides of inequality (as $\widetilde{F}(q, \bar{\alpha}+\beta)=$ $\widetilde{F}(q, \bar{\alpha})+\widetilde{F}(q, \beta)$ and $\widetilde{F}(q, \alpha+\beta)=\widetilde{F}(q, \alpha)+\widetilde{F}(q, \beta)), \widetilde{F}(q, \bar{\alpha})>\widetilde{F}(\alpha, q)$. Thus there is $t \in \alpha$ and such that $\widetilde{F}(q, \bar{t})>\widetilde{F}(t, q)$ and so, by the definition of $\widetilde{N}, q=p_{t}$, for some $p \in P$. Now, it follows from the construction of $\widetilde{N}$, there are $\alpha_{0}, \alpha_{1}, \beta_{0}, \beta_{1}$ and $k \geq 1$ such that $\alpha=t^{k}+\alpha_{0}+\alpha_{1}$ and $\beta=\beta_{0}+\beta_{1}$ and $t \notin \alpha_{0}+\alpha_{1}$ and, for $x=\alpha, \beta$, we have:

$$
\begin{aligned}
& \widetilde{F}\left(x_{1}, p_{t}\right)=\widetilde{F}\left(p_{t}, x_{0}\right)=0=\widetilde{F}\left(p_{t}, \bar{x}_{1}\right)=\widetilde{F}\left(\bar{x}_{0}, p_{t}\right) \\
& \widetilde{F}\left(p_{t}, \bar{x}_{0}\right)=\widetilde{F}\left(x_{0}, p_{t}\right) \quad \widetilde{F}\left(p_{t}, x_{1}\right)=\widetilde{F}\left(\bar{x}_{1}, p_{t}\right) .
\end{aligned}
$$

By $S E Q$ of reachability graphs,

$\phi(M) \stackrel{\alpha_{1}+\beta_{1}}{\longrightarrow} \widetilde{N} \phi(M)+e f \widetilde{N}_{\widetilde{N}}\left(\alpha_{1}+\beta_{1}\right) \stackrel{t^{k}}{\longrightarrow} \widetilde{N} \phi(M)+e f f_{\widetilde{N}}\left(\alpha_{1}+\beta_{1}+t^{k}\right)$.

Thus, by (14), $\phi(M)+e f f_{\widetilde{N}}\left(\alpha_{1}+\beta_{1}+t^{k}\right) \stackrel{\bar{t}^{k}}{\longrightarrow} \widetilde{N} \phi(M)+\operatorname{eff}_{\widetilde{N}}\left(\alpha_{1}+\beta_{1}\right)$, and so

$$
\begin{aligned}
& \phi(M)\left(p_{t}\right)+e f f_{\widetilde{N}}\left(\alpha_{1}+\beta_{1}+t^{k}\right)\left(p_{t}\right) \\
& =\phi(M)\left(p_{t}\right)+\text { eff }_{\widetilde{N}}\left(t^{k}\right)\left(p_{t}\right)+\text { eff }_{\widetilde{N}}\left(\alpha_{1}+\beta_{1}\right)\left(p_{t}\right) \\
& =\phi(M)\left(p_{t}\right)+e^{2} f_{\widetilde{N}}\left(t^{k}\right)\left(p_{t}\right)-F\left(p_{t}, \alpha_{1}+\beta_{1}\right)+F\left(\alpha_{1}+\beta_{1}, p_{t}\right) \\
& =\phi(M)\left(p_{t}\right)+e f f_{\widetilde{N}}\left(t^{k}, p_{t}\right)-F\left(p_{t}, \alpha_{1}+\beta_{1}\right) \geq \widetilde{F}\left(p_{t}, \bar{t}^{k}\right) \text {. }
\end{aligned}
$$


We therefore have: $M^{\prime}\left(p_{t}\right)=M\left(p_{t}\right)+$ eff $\widetilde{N}\left(t^{k}\right)\left(p_{t}\right)-\widetilde{F}\left(p_{t}, \alpha_{1}\right)+\widetilde{F}\left(\alpha_{0}, p_{t}\right) \geq_{\text {by }(16)}$ $\widetilde{F}\left(p_{t}, \bar{t}^{k}\right)+\widetilde{F}\left(p_{t}, \beta_{1}\right)+\widetilde{F}\left(\alpha_{0}, p_{t}\right)=\widetilde{F}\left(p_{t}, \bar{t}^{k}\right)+\widetilde{F}\left(p_{t}, \beta_{1}\right)+\widetilde{F}\left(p_{t}, \bar{\alpha}_{0}\right)=\widetilde{F}\left(p_{t}, \bar{\alpha}\right)+$ $\widetilde{F}\left(p_{t}, \beta\right)=\widetilde{F}\left(p_{t}, \bar{\alpha}+\beta\right)$,

yielding a contradiction with (15). Thus $M^{\prime} \stackrel{\bar{\alpha}+\beta}{\longrightarrow} \widetilde{N}$ holds. By $M^{\prime} \stackrel{\bar{\alpha}+\beta}{\longrightarrow} \widetilde{N}$ and $(B)$ we have:

$$
M^{\prime}+\operatorname{eff}_{\widetilde{N}}(\bar{\alpha}+\beta)=\phi(M)+\operatorname{eff}_{\widetilde{N}}(\alpha)+\operatorname{eff}_{\widetilde{N}}(\bar{\alpha}+\beta)=\phi(M)+e f f_{\widetilde{N}}(\beta) .
$$

We now conclude, by $(14),(A)$, and $(B)$, that $S T S^{\text {mrev }} \simeq_{\phi \circ \psi} C R G_{\widetilde{N}}$.

Finally, if all the steps labelling the arcs of STS are sets, then we can construct a new net $\widetilde{N}^{\prime}$, adding to $\widetilde{N}$ a fresh set of places $P^{\prime}=\left\{p_{t u} \mid t \in T \wedge u \in \bar{T}\right\}$, where each $p_{t u}$ is such that $\widetilde{M}_{0}\left(p_{t u}\right)=1$ and has exactly the following connections $\widetilde{F}\left(t, p_{t u}\right)=\widetilde{F}\left(p_{t u}, t\right)=\widetilde{F}\left(u, p_{t u}\right)=\widetilde{F}\left(p_{t u}, u\right)=1$.

Such places ensure that each step enabled at a reachable marking of $\widetilde{N}$ is a subset of $T$ or a subset of $\bar{T}$. Moreover, the enabling of such steps is not affected by adding $P^{\prime}$, so that in this case we get indeed $S T S^{\text {rev }} \simeq C R G_{\widetilde{N}^{\prime}}$.
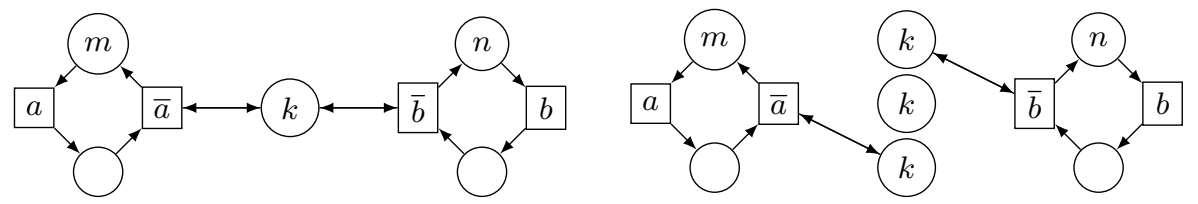

Fig. 6. Net $N_{n, m}$ with $k=\max (m, n)$ and $m, n \geq 1$ (left), and the same net after applying the construction from Theorem 3 (right).

Example 3. Figure 6 depicts a family $N_{n, m}$ of PT-nets which satisfy the assumptions of the last theorem. We clearly have $C R G_{N_{n, m}} \neq S T S^{\text {mrev }}$, where STS is the reachability graph of the net obtained from $N_{n, m}$ by deleting $\bar{a}$ and $\bar{b}$. However, the construction from the proof of Theorem 3 yields the PT-net $C R G_{\widetilde{N}_{n, m}}$ satisfying $C R G_{\widetilde{N}_{n, m}} \simeq S T S^{m r e v}$.

\section{Concluding remarks}

In this paper we conducted what is to the best of our knowledge the first study of reversibility in the $\mathrm{P} / \mathrm{T}$-net model, when the step semantics, based on executing steps (multisets) of actions rather than single actions is considered, thus capturing real parallelism. In a quite more abstract setting, the (partial) reversal of steps, thus generating mixed steps possibly containing both original and reversed events, has been previously studied in [18], but now we are seeing here when and how the reversal can be really done in a concrete operational framework, as Petri Nets are.

Among the topics for future research we would single out an investigation of the impact of allowing multiple reverses of a given action (splitting reverses). Such an idea has already been applied in the case of sequential transition systems, making some non-reversible transition system reversible. 
Acknowledgement. This research was supported by Cost Action IC1405. The first author was partially supported by the Spanish project TRACES (TIN201567522-C3-3), and by Comunidad de Madrid as part of the program S2018/TCS4339 (BLOQUES-CM) co-funded by EIE Funds of the European Union.

\section{References}

1. E. Badouel and Ph. Darondeau. Theory of regions. In LNCS 1491, pages 529-586, 1996.

2. K. Barylska, E. Best, E. Erofeev, Ł. Mikulski, and M. Piątkowski. Conditions for Petri net solvable binary words. ToPNoC, 11:137-159, 2016.

3. K. Barylska, E. Erofeev, M. Koutny, E. Mikulski, and M. Piątkowski. Reversing transitions in bounded Petri nets. volume 157, pages 341-357, 2018.

4. K. Barylska, M. Koutny, Ł. Mikulski, and M. Piątkowski. Reversible computation vs. reversibility in Petri nets. Sci. Comput. Program., 151:48-60, 2018.

5. L. Cardelli and C. Laneve. Reversible structures. In Proc. of CMSB'11, pages 131-140, 2011

6. M.E. Cohen. Systems for financial and electronic commerce, September 32013. US Patent 8,527,406.

7. V. Danos and J. Krivine. Reversible communicating systems. In Proc. of CONCUR'04, volume 3170 of $L N C S$, pages 292-307. 2004.

8. V. Danos and J. Krivine. Transactions in RCCS. In Proc. of CONCUR'05, volume 3653 of $L N C S$, pages 398-412. 2005.

9. V. Danos, J. Krivine, and P. Sobocinski. General reversibility. Electr. Notes Theor. Comp. Sci., 175(3):75-86, 2007.

10. Ph. Darondeau, M. Koutny, M. Pietkiewicz-Koutny, and A. Yakovlev. Synthesis of nets with step firing policies. Fundam. Inform., 94(3-4):275-303, 2009.

11. D. de Frutos-Escrig, M. Koutny, and Ł. Mikulski. An efficient characterization of Petri net solvable binary words. In Proc. of ATPN'18, pages 207-226, 2018.

12. E. Erofeev, K. Barylska, Ł. Mikulski, and M. Piątkowski. Generating all minimal Petri net unsolvable binary words. In Proc. of PSC'16, pages 33-46, 2016.

13. J. Esparza and M. Nielsen. Decidability issues for Petri nets. BRICS Report Series, 1(8), 1994.

14. T. Hujsa, J.-M. Delosme, and A. Munier Kordon. On the reversibility of live equalconflict Petri nets. In Proc. of ATPN'15, volume 9115 of $L N C S$, pages 234-253, 2015.

15. I. Lanese, C.A. Mezzina, and J.-B. Stefani. Reversing higher-order Pi. In Proc. of CONCUR'10, volume 6269 of $L N C S$, pages 478-493, 2010.

16. H.A. Özkan and A. Aybar. A reversibility enforcement approach for Petri nets using invariants. WSEAS Transactions on Systems, 7:672-681, 2008.

17. I. Phillips and I. Ulidowski. Reversing algebraic process calculi. J. of Log. and Alg. Prog., 73(1-2):70-96, 2007.

18. I. Phillips and I. Ulidowski. Reversibility and asymmetric conflict in event structures. J. of Log. and Alg. Meth. in Prog., 84(6):781-805, 2015.

19. W. Reisig. Understanding Petri Nets - Modeling Techniques, Analysis Methods, Case Studies. 2013

20. A. De Vos. Reversible Computing - Fundamentals, Quantum Computing, and Applications. 2010. 